\title{
Introduction to the special focus topic issue on computational electronics of emerging memory elements
}

\author{
Blanka Magyari-Köpe ${ }^{1}$ · Derek Stewart ${ }^{2}$
}

Published online: 17 November 2017

(C) Springer Science+Business Media, LLC 2017

The quest to replace traditional floating-gate flash with some form of nonvolatile memory has led to intensive research in a wide range of technologies including phase change materials, conductive bridge and vacancy-mediated resistive RAM as well as spin-transfer-torque magnetic RAM and ferroelectric memories. For a wide adoption of any of these technologies, numerous factors such as the programming voltages, programming speeds, "ON/OFF" resistance ratio, power, retention, write/erase operation endurance, compatibility with the standard silicon fabrication platform, and environmental issues need to be considered. To address these challenges, the engineering community is closely collaborating with researchers in materials science, physics and chemistry to find practical solutions for integrated circuit manufacturing. Therefore, the goal of this Special Issue is to bring together these various communities to address the current challenges in the emerging memory field. The invited papers in this issue highlight recent advances in the simulation of memory devices and cover both the fundamental materials science and device physics required to move the discipline forward.

The contenders for emerging memories mentioned above involve technologies of very different character, and for each of them, the memory switching process relies on a unique mechanism. While for resistance change memories (RRAMs) and conductive bridge memories (CBRAMs), the change in resistivity is induced by the motion of charged ionic species, in phase change materials (PCRAMs), the switch in resistance is obtained by a crystalline to amorphous

Blanka Magyari-Köpe

blankamk@stanford.edu

1 Department of Electrical Engineering, Stanford University, Stanford, CA, USA

2 Western Digital, San Jose, CA, USA phase transformation. Oxide-based RAM involves switching cycles driven by oxygen vacancy migration and has been attracting increasingly strong attention due to its simple structure and fab-friendly materials. On the other hand, the atomic switch or CBRAM devices involving metal ion diffusion have benefits for low-power applications. Ongoing interest is also strong for high-endurance and high-speed spin-transfer-torque magnetic memories (STT MRAMs), in which the resistance change is achieved by flipping the spins of electrons. Finally, in recent years the development of $\mathrm{HfO}_{2}$-based ferroelectric films (FeRAMs) with a switching mechanism based on the stabilization of the ferroelectric orthorhombic structure is showing good potential due to great scalability and CMOS compatibility. Applications of these devices will, however, require associated selector devices, and this rapidly evolving domain is reaching the material optimization stage. Furthermore, applications of these emerging memories within the neuromorphic realm have been strongly pursued in the past few years obtaining impressive results and attaining important milestones.

To assess the long-term challenges and opportunities of these memories, advanced modeling techniques at various time and length scales are critical. The nanoscale dimensions of these new nonvolatile memories present significant challenges for physical characterization techniques and interpretation of experimental data. Given the complexity of the memory switching phenomena in these devices, insight into the local electronic structure, atomic configurations of interfaces, electronic and spin transport, ion diffusion and thermal properties can be critical. Therefore, computational simulations can play an integral role in determining the key physical mechanisms underlying these devices and also in optimizing device performance by guiding material selection and device dimensions. 
This Special Issue provides the broader simulation community with a snapshot of the diverse simulation research and development activities on emerging memories. The invited papers present the most recent developments in the international community on modeling the nonvolatile memory devices and cover all aspects from first principles based quantum mechanical models, classical molecular dynamics, kinetic Monte Carlo, device-level physical models to compact and circuit models.

The "Computational Electronics of Emerging Memory Elements" Special Issue consist of 21 invited papers giving general reviews and contributions covering the emerging memory spectra. The issue starts off with two papers on $a b$ initio simulations for PCRAM devices, the first addressing thermal conductivity calculations by Emanuele Bosoni et al. (Universita degli Studi di Milano-Bicocca, Italy) and the second covering the topic of thermal boundary resistance by Silvia Gabardi et al. (Universita degli Studi di Milano-Bicocca, Italy). On CBRAM memories, a wide spectrum ranging from fundamental atomistic analysis by Sergiu Clima et al. (imec, Belgium) to a comprehensive multiscale approach by Stephan Menzel (Forschungszentrum Julich, Germany) is presented. Covering resistive memories, first a detailed account is given in terms of fundamental understanding of oxygen vacancy formation by Sebastian Wicklein (Western Digital, USA), followed by a mechanism discussion based on ab initio simulations and experimental insights by Boubacar Traore et al. (Institut des Sciences Chimiques de Rennes, France). Then, the attention is shifted toward elucidating at the atomistic level, the role of electrodes in a paper by Ze-Han Wu et al. (Huazhong University of Science and Technology, China) and characterizing the electron transport through RRAM devices by Xiaoliang Zhong et al. (Argonne National Laboratory, USA). Moving towards larger length and time scales within various device-level approaches, we start with a multiscale description by Luca Larcher and Andrea Padovani (MDLab, Italy), and extend into microand macro analysis proposed by Gennadi Bersuker et al.
(Aerospace Corporation and National Institute of Standards and Technology, USA) and by Marco Villena et al. (Soochow University, China). Switching to TCAD and compact models for RRAM devices, Daniele Ielmini and Valerio Milo (Politecnico di Milano, Italy) address the link between the underlying physical models and neuromorphic applications. Continuing on the neuromorphic topic, John Niroula et al. (Sandia National Laboratories, USA) present an empirically derived general purpose compact model and Stefano Brivio and Sabina Spiga (CNR-IMM, Italy) describe a stochastic circuit breaker network model. Circuit simulations for the cross-point architecture are further addressed by Shimeng $\mathrm{Yu}$ et al. (Arizona State University, USA), and the second part of this paper sets the stage for the selector device component discussion. A new threshold device design based on a field triggered thermal runaway process is addressed in a paper by Carsten Funck et al. (Institut für Werkstoffe der Elektrotechnik Aachen, Germany). Concluding on the selector topic, An Chen (SRC, USA) presents a comprehensive review on the performance requirements and device options for crossbar arrays. In the STT MRAM domain, a detailed review on the current stages of spin-transfer torque-based magnetic memories is given by Yunkun Xie and co-authors (University of Virginia, USA). For the recently proposed $\mathrm{HfO}_{2}$-based ferroelectric devices, fundamental aspects related to phase stability are addressed by Sergey Barabash (Intermolecular, USA), followed by a multiscale assessment from atomistic to circuit level given by Milan Pesic et al. (NaMLab, Germany). Finally, concluding the Focus Topic Issue, a generic review on compact models for PCRAM, STT-MRAM and RRAM memories coupling physical equations from multiple domains is presented by Nuo Xu (Samsung, USA).

We hope that the readers will enjoy the invited papers and that this issue will serve as a comprehensive resource for academic, national laboratory and industry professionals. We would like to express our sincere appreciation to all the authors of the invited papers submitted to this Special Issue. 